\title{
EAl Endorsed Transactions

\section{Experimental analysis of intelligent vehicle monitoring system using Internet of Things (IoT)}

\author{
T.Thamaraimanalan ${ }^{1, *}$, M.Mohankumar ${ }^{1}$, S.Dhanasekaran ${ }^{1}$ and H.Anandakumar ${ }^{2}$ \\ ${ }^{1}$ Assistant Professor, Department of ECE, Sri Eshwar College of Engineering, Coimbatore. \\ ${ }^{2}$ Associate Professor, Department of CSE, Sri Eshwar College of Engineering, Coimbatore.
}

\begin{abstract}
Internet of things is an emerging trend and Wireless sensor networks are embedded with IoT to measure various parameters of a vehicle. Measurements and standards of measurements are the most important stages for measuring an object's size, length, weight etc., Measuring the weight of small objects does not need much time and efforts, but on the other hand weighing large objects needs lots of time and efforts. A new approach is proposed to monitor the location of the vehicle, engine temperature, tyre pressure, oil level, speed control and load measurement of a vehicle. Even if the device is offline, the logs will be stored in the memory card and it can be used for future analysis. In addition to this theft control is introduced for the security of the vehicle. CC3200 microcontroller is connected with load cell to monitor the total weight of the vehicle and engine temperature is monitored using the temperature sensor. By GPS the location and speed of the vehicle is monitored periodically and the location is uploaded in the cloud environment. Vehicle monitoring with load calculation, anti-theft control and the data acquisition from various sensors incorporated with the Electronic Control Unit (ECU) is collected and processed in the cloud. The proposed framework is effectively adaptable at low cost.
\end{abstract}

Keywords: Data acquisition, Internet of Things (IoT), Vehicle monitoring system.

Received on 04 February 2021, accepted on 08 April 2021, published on 16 April 2021

Copyright (C) 2021 T.Thamaraimanalan et al., licensed to EAI. This is an open-access article distributed under the terms of the Creative Commons Attribution license, which permits unlimited use, distribution, and reproduction in any medium so long as the original work is properly cited.

doi: 10.4108/eai.16-4-2021.169336

*Corresponding author. Email:t.thamaraimanalan@gmail.com

\section{Introduction}

In the early days' individuals utilized various procedures to quantify a specific item. To begin with, they used to figure the loads of an item by utilizing their hands to gauge. After that utilization of equilibriums came to presence to gauge the objects. Even though different types of balances came into existence, still the exact standard of measurement is in debate and it differs from one part of the world to another part of the world. As measurement is the process of measuring a particular object relative to the predetermined measurement standards and these standards has to be accurate and reliable. Nowadays Weigh Bridge is used to weigh heavy objects or goods, but the main consideration to use this weigh bridge is the consumption of time and energy.

Vehicular thefts and unauthorized vehicular usage within a locality are of major concern that leads to vehicle monitoring. Until today, research was carried on vehicle monitoring using GPS/GPRS techniques.

Usually, the weigh bridge is located at a distance from the loading place and to measure the goods one has to travel to the place where the weigh bridge is located which takes time and energy. If the material which is there in the logistics to transport is costlier, then there is a necessity to track the vehicle also certain other parameters such as speed, fuel, temperature have to be monitored. Figure 1 shows the details about cloud access with various digital gadgets. 


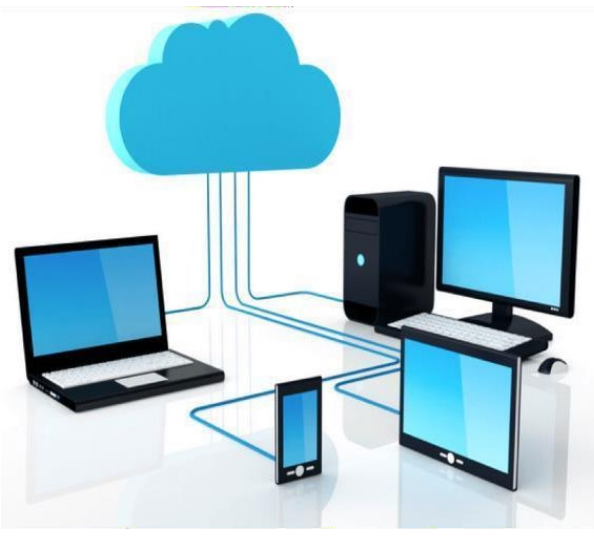

Figure 1. Cloud Access

\section{Related Work}

Branisso, L. B, et al., [1] proposed a vehicle monitoring system using GPS, GSM, and GIS. In this system, the PC is used as base-station and the number of vehicles using this system is limited. Only location monitoring is taken into consideration.

Muller I, et al [7] implemented a system using to measure the load of the vehicle using load cell. The theoretical details are shared for application purpose and it is not implemented using any hardware.

Tan, et al [10] developed an Android-based application that detects an accidental situation and sends an alert message to the nearest police station and medical care centre. This application is organized with an external pressure sensor to extract the outward force of the vehicle body. Hence, the application plays an important role in Postaccident services.

Harrison $G G$, et al [2] proposed a system that has an Android mobile assembled with GPS and GSM modules along with a processor that is setup in the vehicle. During the movement of the vehicle, the location of the vehicle is continuously observed in the web server using GPRS.

$\mathrm{Li}$, et al [7] suggested a framework that is based on Raspberry pi that is connected to $3 \mathrm{G} / 4 \mathrm{G}$ dongle used as a Modem. The vehicle unit is attached to the vehicle, the attached unit is configured to receive signals from a mobile tower and send it to web server to represent the location on the map in the real time.

Sasvanth Narayan, et al [9] designed and implemented vehicle tracking, vehicle monitoring, and controlling and vehicle status. In this system vehicle door, parking lights, side mirrors are monitored and controlled by a mobile phone.

\section{Proposed Work}

\subsection{Hardware and Software description}

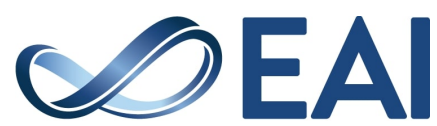

The applications MCU subsystem contains an industrystandard ARM Cortex-M4 core running at $80 \mathrm{MHz}$. The device includes a wide variety of peripherals, including a fast parallel camera interface, I2S, SD/MMC, UART, SPI, I2C and four-channel ADC [13]. The CC3200 family includes flexible embedded RAM for code and data and ROM with external serial flash boot loader and peripheral drivers.

Figure 2 shows the Hardware overview of CC3200 controller.

The Wi-Fi network processor subsystem features a Wi-Fi Internet-on-a-Chip and contains an additional dedicated ARM MCU that completely offloads the applications MCU. This subsystem includes an $802.11 \mathrm{~b} / \mathrm{g} / \mathrm{n}$ radio, baseband, and MAC with a powerful crypto engine for fast, secure Internet connections with 256-bit encryption. The CC3200 device supports Station, Access Point, and Wi-Fi Direct modes. The device also supports WPA2 personal and enterprise security and WPS 2.0. The Wi-Fi Internet-on-achip includes embedded TCP/IP and TLS/SSL stacks, HTTP server, and multiple Internet protocols. Figure 3 shows the software overview of the controller.

The power-management subsystem includes integrated DC-DC converters supporting a wide range of supply voltages. This subsystem enables low-power consumption modes, and hibernates with RTC mode requiring less than 4 $\mu \mathrm{A}$ of current.

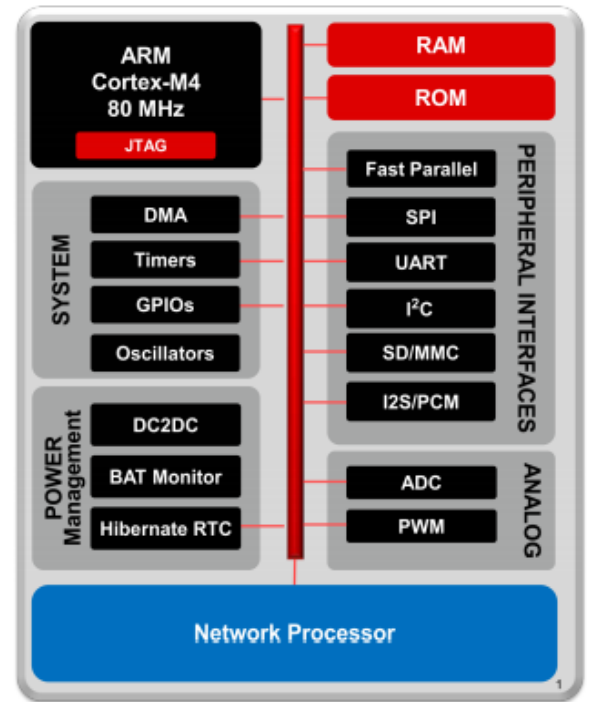

Figure 2. Hardware overview of CC3200 


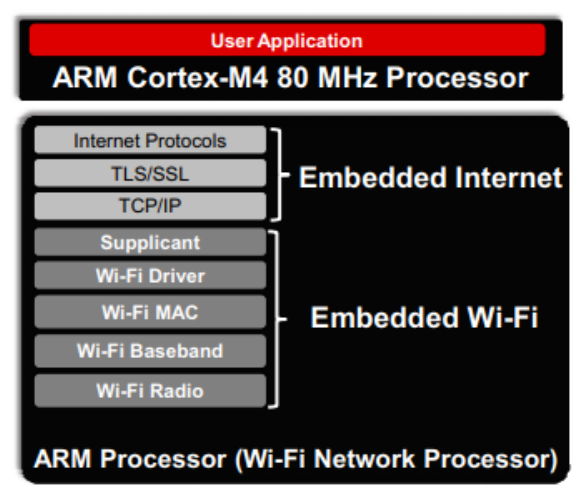

Figure 3. CC3200 Embedded Software Overview

\subsection{Details of the proposed model}

The proposed model is capable of monitoring the following parameters in a heavy load vehicle. Figure 4 shows the block diagram of the proposed model.

(i) From the vehicle wheels, speed of the vehicle, braking system and tire pressure are monitored using Google maps, transducers and pressure sensor respectively.

(ii) Using float sensor, the level is fuel available in the fuel tank is monitored periodically and the details are stored in cloud.

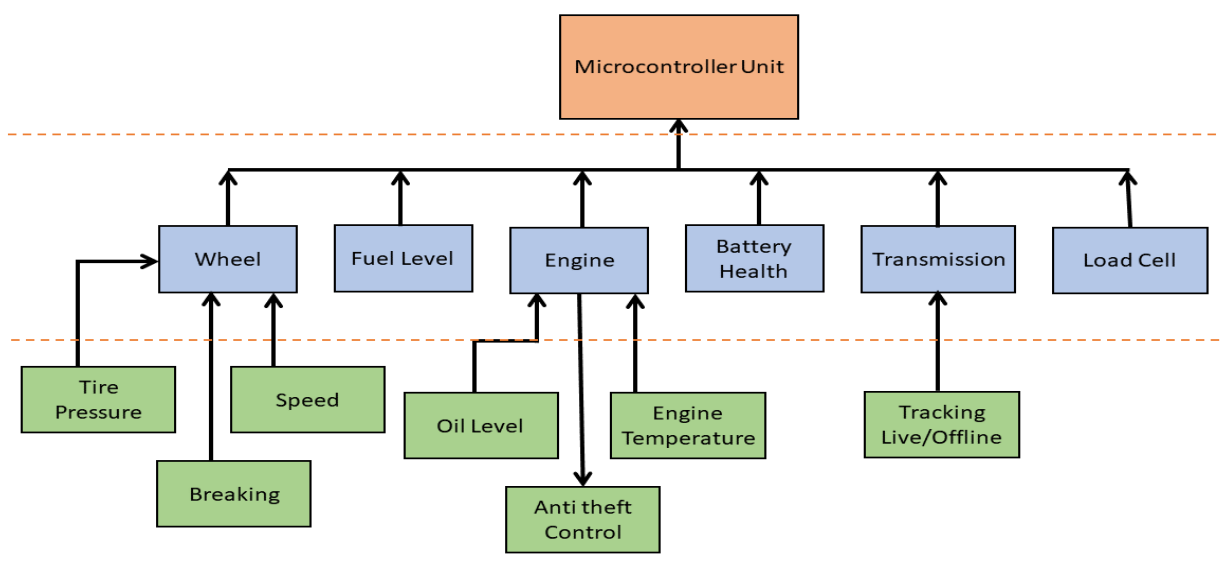

Figure 4. Block diagram of the proposed model

(iii) In Engine, the oil level is monitored from magnetic reed switches which are covered with stainless steel body. The engine temperature is constantly viewed through temperature sensor. A simple 4MP camera is kept in front of the driver seat. Whenever the driver ignites the engine, a image of the driver will be sent to the cloud and in the cloud the image is compared with the authorised available database. If the image is not matched, immediately the engine will be turned off. This ensures the security of the vehicle.

(iv) Always the health of the battery in the vehicle is measured using Monnit Wireless Battery Health Sensors. These sensors are analog voltage meters designed to measure battery voltage at specified intervals and send the voltage readings to iMonnit, the online sensor monitoring system. If the condition of the battery is poor and needs to be replaced, an alert message will be sent to the owner.

(v) The location of the vehicle is monitored through Google maps and for every 5 minutes the location of the vehicle will get updated. Suppose if the vehicle is not in a network coverage area, all the travelling logs will be stored in the memory card. When network connection is established again the logs in the memory will send to the cloud.

(vi) Load cell is used to measure the load vehicle.

The brief overview of the proposed system is given in figure 5 .

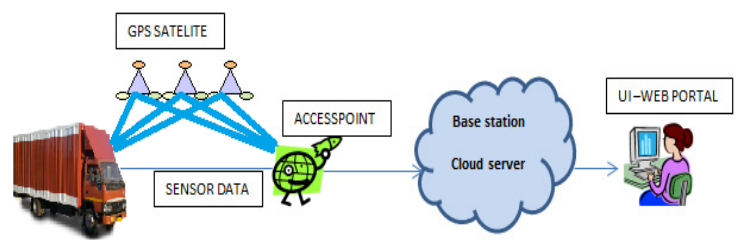

Figure 5. Overview of the proposed system

\section{Results and Discussions}

Various sensors are assigned to monitor the parameters of vehicle and driver details. All the details are transmitted 
to the signal conditioner to convert one type of electronic signal into another type of signal for further process. The ARM processor in the $\mathrm{CC} 3200$ transmits the data to the cloud. The process flow of the intelligent vehicle monitoring system (IVMS) is shown in figure 6 .

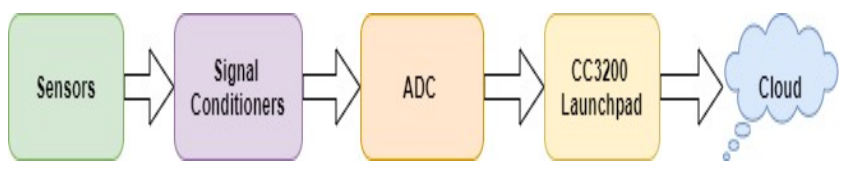

Figure 6. Process flow diagram of the IVMS

Cloud is a platform which provides the logical and physical infrastructure to store the data. Security for the data in the cloud environment is ensured by authorized login. The main advantage of using cloud is, any business can get the benefits of the cloud instantly to access the data anywhere from the globe.
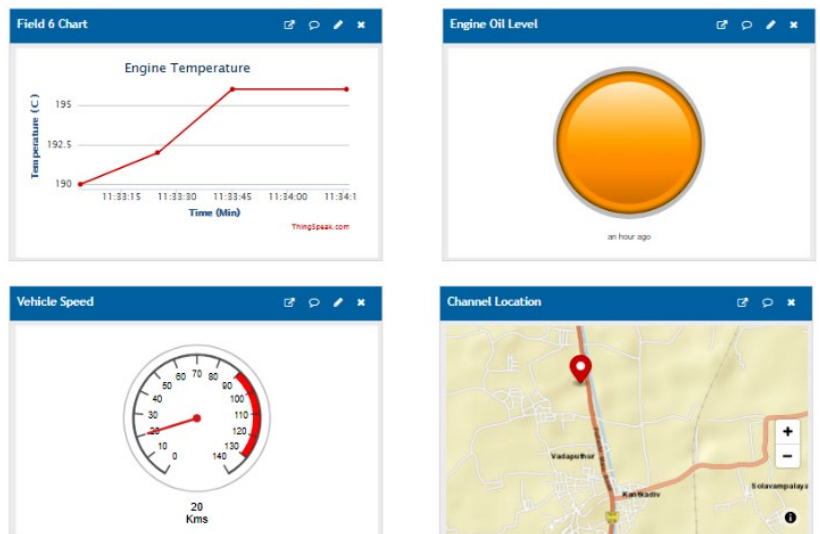

Figure 7(a). Experimental result of Temperature, Engine oil level, Vehicle speed and live location

Figure 7(a) shows the experimental results of Engine temperature, Engine oil level, Speed and location of the vehicle. By default, the engine oil level indicator is in "orange" indication and when oil level is reduced the indicator gives "red" indication. For every 5 minutes of time interval the details will get updated with the refreshed values received from the vehicle.
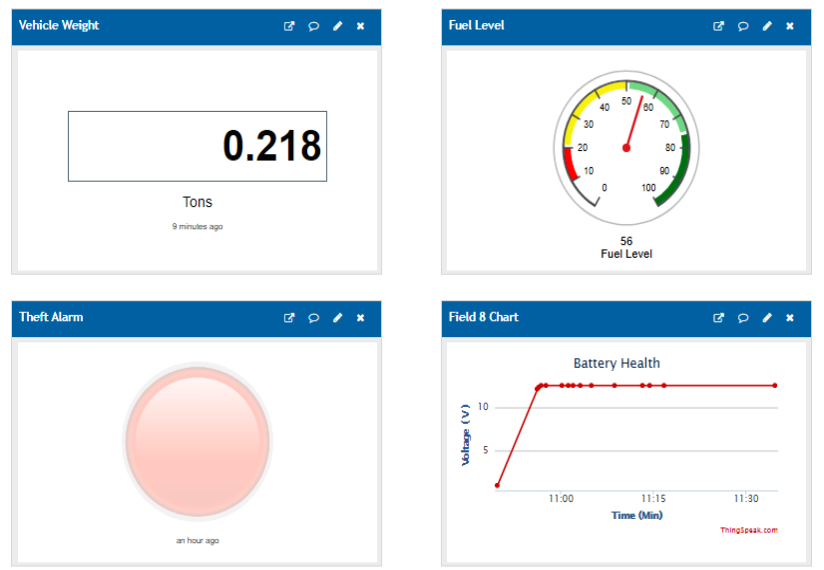

Figure 7(b). Experimental results of Vehicle load, Fuel level, Theft indication and battery health

Figure 7(b) shows the experimental results of vehicle weight, Fuel level, Anti-theft indicator and Battery health. If theft of the vehicle is identified, an alert message will sent to the owner along with the location of the vehicle.

\section{Conclusion}

Experimental analysis of Intelligent Vehicle Monitoring System (IVMS) using Internet of Things (IoT) and Cloud Computing is developed. The proposed is based on cloud computing infrastructure along with sensors useful for monitoring the load weight, fuel level, battery health, tire pressure, anti-theft, engine temperature, engine oil, speed, location of the vehicle and braking system. All the data acquired from the sensors are transferred to cloud server using CC3200. All the data are kept in a single page for easy access. The proposed technology significantly provides the vital information of the vehicle and it is cost efficient.

\section{References}

[1] Branisso, L. B., Kato, E. R. R., Pedrino, E. C., Morandin Jr., O., \& Tsunaki, R. H. (2012). An Intelligent Autonomous Vehicle Management System. 2012 Second Brazilian Conference on Critical Embedded Systems. doi:10.1109/cbsec. 2012.23

[2] Harrison, C. G. (1998). A vehicle management system. IEE Colloquium on Automotive Radar and Navigation Techniques. doi:10.1049/ic:19980194

[3] K Kavitha, T Thamaraimanalan, M Suresh Kumar (2014), An Optimized Heal Algorithm for Hole Detection and Healing in Wireless Sensor Networks International Journal of Advanced Engineering Research and Technology (IJAERT), Vol 2, Issue 3, pp 243-249.

[4] He, H., \& Wang, Y. (2015). Simulation of piezoelectric sensor in weigh-in-motion systems. 2015 Symposium on Piezoelectricity, Acoustic Waves, and Device Applications(SPAWDA).doi:10.1109/spawda.2015.73647

[5] Li, B., Zhan, S., Hu, J., Sun, W., \& Zhang, G. (2018). New Strategy for Weigh-in-Motion Measurement Based on Vibration Sensors. 2018 IEEE 8th Annual International Conference on CYBER Technology in Automation, Control, and Intelligent Systems (CYBER). doi:10.1109/cyber.2018.8688210

[6] Li, Z., Xueping, Y., Yu, W., \& Zhifa, Y. (2013). System of real-time monitoring dynamic vehicle load status. Proceedings of 2013 IEEE International Conference on Vehicular Electronics andSafety. doi:10.1109/icves.2013.6619617

[7] Muller, I., de Brito, R., Pereira, C., \& Brusamarello, V. (2010). Load cells in force sensing analysis -- theory and a novel application. IEEE Instrumentation \& Measurement Magazine, 13(1), 15-19. doi:10.1109/mim.2010.5399212

[8] Sasvanth Narayan, R., Loganathan, V., Lakkar, P., \& Suganthan, B. (2019). IoT Cloud based Optimization of Vehicle Using Monitoring Systems. 2019 International 
Conference on Communication and Electronics Systems (ICCES). doi:10.1109/icces45898.2019.9002241

[9] T.Thamaraimanalan, S.P.Vivekk, G.Satheeshkumar and P.Saravanan (2018), Smart Garden Monitoring System Using IOT, Asian Journal of Applied Science and Technology, Vol 2 (2), Pg 186-192.

[10] Tan, Y., Robotis, A., \& Kanellakopoulos, I. (n.d.). Speed control experiments with an automated heavy vehicle. Proceedings of the 1999 IEEE International Conference on Control Applications. doi:10.1109/cca.1999.801169

[11] S. Yasotha, V. Gopalakrishnan \& M. Mohankumar," Multi-sink Optimal Repositioning for Energy and Power Optimization in Wireless Sensor Networks" in Wireless Personal Communications, Volume 82, Number 3, June(1),2015.

[12] M. Mohankumar, V. Gopalakrishnan and S.Yasotha, "A Vlsi Approach For Distortion Correction In Surveillance Camera Images," in ARPN Journal of Engineering and Applied Sciences, Vol. 10, No. 9, May 2015 ISSN 18196608.

[13] https://www.ti.com/product/CC3200 